\title{
Instability of Spikes in the Presence of Conservation Laws
}

\author{
Alin Pogan and Arnd Scheel \\ University of Minnesota \\ School of Mathematics \\ 206 Church St. S.E. \\ Minneapolis, MN 55455, USA
}

\begin{abstract}
We show that spikes are unstable in a class of scalar reaction-diffusion equations coupled to a general conservation law. Our class includes the Keller-Segel model for chemotaxis, phase-field models and models for chemical reactions in closed chemical reactors. ${ }^{1}$
\end{abstract}

\section{Introduction}

Localized self-organized structures are among the most elementary yet striking phenomena caused by nonlinearity in spatially extended dynamical systems. While such localized structures can be observed in a variety of experimental scenarios, the mathematical problem of proving existence and stability is often quite difficult. Many if not most existence proofs are based in various ways on the fact that a simple scalar reaction diffusion system possesses localized solutions, which we refer to as spikes. The simplest example is the scalar equation

$$
v_{t}=v_{x x}-v+v^{2}, t \geq 0, x \in \mathbb{R}
$$

which possesses the stationary solution $v^{*}(x)=\frac{3}{2} \operatorname{sech}^{2}\left(\frac{x}{2}\right)$.

It was recognized early, however, that spikes, as well as more general, non-monotone solutions are always unstable in scalar reaction-diffusion systems [5].

Our focus here is on an extension of such scalar equations by a conservation law,

$$
\left\{\begin{array}{l}
u_{t}=\left[a(u, v) u_{x}+b(u, v) v_{x}\right]_{x}, \quad t \geq 0, x \in \mathbb{R}, \\
v_{t}=v_{x x}+f(u, v),
\end{array}\right.
$$

with functions $a, b$, and $f$ of class $C^{3}\left(\mathbb{R}^{2}, \mathbb{R}\right)$. In order to ensure well-posedness on appropriate function spaces, we also assume that $a(u, v) \geq a_{0}>0$ for all $(u, v) \in \mathbb{R}^{2}$. We note that the equation for $u$ is a conservation law, that is, $\int u(t, x)$ is independent of time under suitable decay (or boundary) conditions.

The general form of (1.1) incorporates several model problems from chemistry, biology, and material science. We briefly explain here how models for phase separation, precipitation, chemotaxis, and cell polarization can be rewritten in the form (1.1).

\footnotetext{
${ }^{1}$ AMS MSC 2000 Mathematics Subject Classification: 37L15

Keywords: stability, essential spectrum, spikes, conservation law
} 
A simple chemical conversion reaction $A \longleftrightarrow B$ in a closed reactor can often be described by a reaction-diffusion system for the concentrations $u_{1}=[A]$ and $u_{2}=[B]$,

$$
\left\{\begin{array}{c}
u_{1, t}=d_{1} u_{1, x x}-g\left(u_{1}, u_{2}\right), \\
u_{2, t}=d_{2} u_{2, x x}+g\left(u_{1}, u_{2}\right),
\end{array} \quad t \geq 0, x \in \mathbb{R} .\right.
$$

Here, $g$ is the combined reaction rate of the conversion $A \longleftrightarrow B$, and $d_{j}$ are diffusion rates. The simple change of variables $u=u_{1}+u_{2}, v=u_{2}$ and a rescaling of $x$ then immediately transforms (1.2) into (1.1), with constant functions $a$ and $b$. Such conversion reactions have also been used to model precipitation kinetics $[2,7,14]$ that are responsible for the formation of spikes in Liesegang patterns. Here, kinetics $g$ often exhibit a bistable characteristic, for which we give an example in Section 6. Models of this form also arise in biological contexts, for instance in modeling the polarization of motile eukaryotic cells in response to external signals [11].

Seemingly different models arise in the theory of thermodynamic phase transitions. Consider for instance the phase-field system

$$
\left\{\begin{array}{l}
\tau \varphi_{t}=d_{\varphi} \varphi_{x x}+\frac{1}{2}\left(\varphi-\varphi^{3}\right)+2 w, \\
w_{t}+\frac{1}{2} l \varphi_{t}=d_{w} w_{x x}
\end{array} \quad t \geq 0, x \in \mathbb{R}\right.
$$

where $w$ denotes temperature and the phase function $\varphi$ acts as an order parameter, so that $\varphi=-1$ corresponds to the solid phase and $\varphi=1$ to the liquid phase. The constant $d_{w}$ characterizes heat flux, the constant $d_{\varphi}$ accounts for finite interfacial energy, and $l$ is the latent heat; see [1]. Again, a simple transformation, $u=w+\frac{l}{2} \varphi, v=\varphi$, and a rescaling of space and time, converts the phase-field system (1.3) into the conservation law form (1.1). A quite common, related model for phase separation is the Cahn-Hilliard equation

$$
w_{t}=-\left(w_{x x}+w-w^{3}\right)_{x x}, t \geq 0, x \in \mathbb{R},
$$

which in fact shares many common features with our class of models (1.1), even if it cannot be cast in the exact same form. We comment briefly on the applicability of our methods to this equation in Section 6.

Slightly more general functions $a$ and $b$ arise in chemotaxis models, where $u$ measures the concentration of a cell population, and $v$ the concentration of a chemical produced by the bacteria. Motility of bacteria may depend on concentrations, and direction of motion on gradients of the chemical. The chemotactic behavior is typically encoded in a function $b(u, v)=-u k(u, v)$, with chemotactic sensitivity $k>0$. The function $f$ in (1.1) models concentration-dependent production and degradation of the chemical $v$. The simplest case, $a \equiv 1, k \equiv \chi$, and $f(u, v)=u-v$ is the classical Keller-Segel model [13]; see [8] for an overview and [9] for specific examples of spike formation.

Spikes can be found theoretically in all the models mentioned above. They have also been observed both numerically and experimentally; see for instance [2, 7, 13, 14]. Also, while experiments are set in finite domains, it is common that the domain size is large compared to characteristic length scales of patterns, so that the influence of boundary conditions often seems negligible. 
In order to see why spikes are ubiquitous in all those models, one solves the steady-state equation first for $u$, and then for $v$. The equation in (1.1) for $u$ can be integrated once and then transformed into a differential equation for $u$ in terms of the independent variable $v$,

$$
\frac{\mathrm{d} u}{\mathrm{~d} v}=-\frac{b(u, v)}{a(u, v)} .
$$

One then proceeds and substitutes the solution $u=\Phi(v)$ into the equation for $v$ and finds

$$
v_{x x}+f(\Phi(v), v)=0 .
$$

Under suitable conditions on $f$ and $\Phi$, this equation will possess homoclinic solutions $v(x)=$ $v(-x) \rightarrow v_{\infty}$ as $x \rightarrow \infty$, which yield spikes for the full system via $u(x)=\Phi(v(x))$.

We are interested in spikes that are asymptotic to solutions that are stable for the pure kinetics, that is, $\left(u^{\infty}, v^{\infty}\right)$ is a stable equilibrium to

$$
\left\{\begin{array}{l}
u^{\prime}=0 \\
v^{\prime}=f(u, v),
\end{array}\right.
$$

which is ensured by the open assumption that $f_{v}\left(u^{\infty}, v^{\infty}\right)<0$.

Our main result asserts that spike solutions to any of the above models are always unstable when considered as solutions on the unbounded real axis, $x \in \mathbb{R}$. As a corollary, we obtain that any given spike is unstable when considered on a sufficiently large domain with, say, Neumann boundary conditions.

More precisely, we have the following result.

Theorem 1.1. Suppose (1.1) possesses a spike solution $\left(u^{*}, v^{*}\right)$, that is,

$$
\left|\left(u^{*}-u^{\infty}, v^{*}-v^{\infty}\right)(x)\right| \leq C \mathrm{e}^{-\eta|x|}, \quad\left(u^{*}, v^{*}\right) \not \equiv\left(u^{\infty}, v^{\infty}\right),
$$

for some constants $u^{\infty}, v^{\infty}$, and $C, \eta>0$. Moreover, assume that $f_{v}\left(u^{\infty}, v^{\infty}\right) \neq 0$.

Then $\left(u^{*}, v^{*}\right)$ is unstable as an equilibrium to (1.1), considered as an evolution equation on the space of bounded uniformly continuous functions $B U C\left(\mathbb{R}, \mathbb{R}^{2}\right)$.

Remark 1.2. We comment on some variations of this main result.

(i) Our result immediately gives instability of spikes in the Keller-Segel model and bistable precipitation models.

(ii) We will outline in the discussion how this result yields instability of spikes in sufficiently large but bounded domains.

(iii) The actual result is sharper, as we will see from the proof. It guarantees the existence of a real positive eigenvalue to the linearization, provided that the background state $\left(u^{\infty}, v^{\infty}\right)$ is linearly stable in an appropriate sense. As a consequence, perturbations of spikes typically grow exponentially. 
(iv) Instability results also hold in most other common functions spaces, such as $L^{p}$ - or $L_{\text {unif }}^{p}$ spaces.

Our approach is as follows. First we linearize the system (1.1) along the spike $\left(u^{*}, v^{*}\right)$. Next, we compute the essential spectrum of the linearized operator $\mathcal{L}$, using a compact perturbation argument similar to the approach given in [6]. In the case when the essential spectrum is stable, we trace the point spectrum of $\mathcal{L}$ by using a homotopy to an easier system. The homotopy is constructed such that at the end of the homotopy interval the system has exactly one positive eigenvalue. Furthermore, we show that the eigenvalues of $\mathcal{L}$ stay away from 0 and that a parity index is constant during the homotopy. One major difficulty of the proof is that $0 \in \sigma_{\text {ess }}(\mathcal{L})$. To bypass this problem we show that $\mathcal{L}$ is a Fredholm operator with negative index on exponentially weighted spaces, which allows us to use the Lyapunov-Schmidt reduction method to solve the eigenvalue problem for $\mathcal{L}$. An alternate method to track point spectrum near the edge of the essential spectrum, where $\mathcal{L}$ ceases to be Fredholm of index 0, would use the Evans function and the Gap Lemma, see e.g., [3, 12]. Our approach is in some sense more direct but less geometric.

The paper is organized as follows. We prove the existence of a smooth family of spikes and derive a hyperbolicity condition in Section 2. Section 3 formulates a spectral instability result and shows how this implies our main result. We then characterize parameter values for which the essential spectrum is stable in Section 4. The core of our argument is contained in Section 5 , where we construct homotopies and trace the point spectrum. We conclude with a few more explicit examples, Section 6, and a brief discussion, Section 7.

Notations: We collect some notation that we will use throughout this paper. For an operator $T$ on a Hilbert space $X$ we use $T^{*}, \operatorname{dom}(T), \operatorname{ker} T, \operatorname{im} T, \sigma(T), \rho(T)$ and $T_{\mid Y}$ to denote the adjoint, domain, kernel, range, spectrum, resolvent set and the restriction of $T$ on a subspace $Y$ of $X$. If $g: \mathbb{R}^{2} \rightarrow \mathbb{R}$ is a smooth function, e.g., $a, b, f$ or one of its partial derivatives, we denote by $g^{*}:=g\left(u^{*}, v^{*}\right)$ and $g^{\infty}:=g\left(u^{\infty}, v^{\infty}\right)$.

Acknowledgement. The authors gratefully acknowledges support by the National Science Foundation under grant NSF-DMS-0806614.

\section{Families of spikes}

In this section, we show that the assumption on existence of an exponentially localized spike actually implies the existence of a family of spikes. We therefore consider the steady-state equation

$$
\left\{\begin{array}{l}
{\left[a(u, v) u_{x}+b(u, v) v_{x}\right]_{x}=0} \\
v_{x x}+f(u, v)=0 .
\end{array}\right.
$$

First note that the spike is a bounded solution, so that we may without loss of generality assume that the function $b(u, v)$ is bounded, possibly modifying $b$ for large values of $u$ and $v$. In the sequel, we therefore always assume that $b$ is bounded.

Next, notice that the convergence of $u^{*}$ and $v^{*}$ for $x \rightarrow \pm \infty$ implies that $u_{x}^{*}$ and $v_{x}^{*}$ converge 
to zero. To see this, note that the second equation in (2.1) implies that $v_{x x}^{*}$ is bounded, which together with Taylor's formula implies that $v_{x}^{*}$ converges to zero. Next, we integrate the first equation in (2.1) and find $a(u, v) u_{x}+b(u, v) v_{x}=m$ for some $m \in \mathbb{R}$. Since $u^{*}, v^{*}, v_{x}^{*}$ converge as $x \rightarrow \pm \infty$, we conclude that $u_{x}$ converges and the limit must vanish since $u^{*}$ is bounded. As a consequence, we find that a spike necessarily satisfies the ODE (1.4),

$$
\frac{\mathrm{d} u}{\mathrm{~d} v}=-\frac{b(u, v)}{a(u, v)}, \quad u\left(v_{0}\right)=u_{0} .
$$

Since $b$ is bounded and $a$ bounded away from zero, this ODE possesses a global, smooth solution taking initial conditions $u_{0}$ at $v=v_{0}$ to the solution at $v$,

$$
u(v)=\Phi\left(v, v_{0} ; u_{0}\right), \quad \Phi\left(v_{0}, v_{0} ; u_{0}\right)=u_{0} .
$$

For our particular spike $\left(u^{*}, v^{*}\right)$, we can normalize at infinity, which gives

$$
u^{*}=\Phi\left(v^{*}, v^{\infty} ; u^{\infty}\right)=: \varphi_{0}\left(v^{*}\right) .
$$

We may now substitute the relation $\varphi_{0}$ between $u$ and $v$ into the second-order equation for $v$, which gives the second-order differential equation for $v^{*}$,

$$
v_{x x}+H(v)=0, \quad H(v):=f\left(\varphi_{0}(v), v\right) .
$$

Note that from this ODE alone we can conclude that the spike is an even function, $u^{*}(x)=$ $u^{*}(-x), v^{*}(x)=v^{*}(-x)$, after possibly shifting in $x$.

Exponential convergence of $v^{*}(x)$ for $x \rightarrow \pm \infty$ implies that $H^{\prime}\left(v^{\infty}\right)<0$. We refer to this condition, implied by exponential convergence of the spike, later on as

\section{ODE-Hyperbolicity:}

$$
H^{\prime}\left(v^{\infty}\right)=f_{v}^{\infty}-\frac{b^{\infty}}{a^{\infty}} f_{u}^{\infty}<0
$$

The next lemma guarantees the existence of a smooth family of spikes.

Lemma 2.1. Under the assumptions of Theorem 1.1, there is $\varepsilon>0$ and a family of spikes $\left(u^{*}(\cdot, \mu), v^{*}(\cdot, \mu)\right)$ for $\mu \in(-\varepsilon, \varepsilon)$, such that

(i) the asymptotic values $\left(u^{\infty}(\mu), v^{\infty}(\mu)\right)$ are smooth functions and $0 \neq \partial_{\mu}\left(u^{\infty}(\mu), v^{\infty}(\mu)\right)$; moreover, if $f_{v}^{\infty} \neq 0$ then $\partial_{\mu} u^{\infty}(0) \neq 0$;

(ii) the spikes $\left(u^{*}(\cdot, \mu)-u^{\infty}(\mu), v^{*}(\cdot, \mu)-v^{\infty}(\mu)\right)$ are given as smooth maps from $(-\varepsilon, \varepsilon)$ into $H_{\text {even }}^{2}\left(\mathbb{R}, \mathbb{R}^{2}\right)$; moreover, $\left(u^{*}(\cdot, 0), v^{*}(\cdot, 0)\right)=\left(u^{*}(\cdot), v^{*}(\cdot)\right)$.

Proof. Since $\left(u^{*}, v^{*}\right)$ is a spike in (1.1) we have $v_{x x}^{*}+f\left(u^{*}, v^{*}\right)=0$, which implies $f\left(u^{\infty}, v^{\infty}\right)=$ 0 . We first construct the family $\left(u^{\infty}(\mu), v^{\infty}(\mu)\right)$ and in a second step construct spikes which actually asymptote to these values. From ODE-Hyperbolicity, (2.4), it follows that $f_{u}^{\infty} \neq 0$ or $f_{v}^{\infty} \neq 0$. We may therefore solve $f(u, v)=0$ locally near $u^{\infty}, v^{\infty}$ and denote the solution by $\left(u^{\infty}(\mu), v^{\infty}(\mu)\right), \mu \in(-\varepsilon, \varepsilon)$, and we may assume that

$$
\partial_{\mu}\left(u^{\infty}(0), v^{\infty}(0)\right)=\left(-f_{v}^{\infty}, f_{u}^{\infty}\right) .
$$


We next define

$$
\Phi^{\mu}: \mathbb{R} \rightarrow \mathbb{R}, \quad \Phi^{\mu}(v)=\Phi\left(v, v^{\infty}(\mu) ; u^{\infty}(\mu)\right)
$$

and

$$
\tilde{H}: \mathbb{R} \times(-\varepsilon, \varepsilon) \rightarrow \mathbb{R}, \quad \tilde{H}(v, \mu)=f\left(\Phi^{\mu}(v), v\right) .
$$

With these definitions, clearly $\tilde{H}\left(v^{\infty}(\mu), \mu\right)=0$, and spikes are solutions to

$$
v_{x x}+\tilde{H}(v, \mu)=0,
$$

with $u=\Phi^{\mu}(v)$. Again, by ODE-Hyperbolicity $(2.4), \tilde{H}_{v}\left(v^{\infty}, 0\right)=H^{\prime}\left(v^{\infty}\right)<0$. In particular, $v^{\infty}(\mu)$ is the locally unique equilibrium to $(2.6)$, and $v^{\infty}$ is hyperbolic.

From here, one can obtain a smooth family of spikes using simple phase plane analysis for (2.6). Here, we give a short argument using the Implicit Function Theorem. We view (2.6) as an equation for $w=v-v^{\infty}(\mu)$, which gives a nonlinear operator equation

$$
G(w, \mu)=w_{x x}+\tilde{H}\left(w(\cdot)+v^{\infty}(\mu), \mu\right)=0
$$

where the nonlinearity vanishes at the origin so that $G: H_{\text {even }}^{2}(\mathbb{R}) \times(-\varepsilon, \varepsilon) \rightarrow L_{\text {even }}^{2}(\mathbb{R})$ is a smooth map. Using the definition of $H$ and the fact that $\left(u^{*}, v^{*}\right)$ is a spike in (1.1), one readily verifies $G\left(v^{*}-v^{\infty}, 0\right)=v_{x x}^{*}+H\left(v^{*}\right)=0$. The linearization of $G$ in this solution is

$$
\mathcal{G}=\left(G_{w}\left(v^{*}-v^{\infty}, 0\right)\right) w=w_{x x}+\tilde{H}_{v}\left(v^{*}(\cdot), 0\right) w .
$$

Next, we will show that $G_{w}\left(v^{*}-v^{\infty}\right)$ is invertible. We may consider $\mathcal{G}$ naturally as an operator from $H^{2}(\mathbb{R})$ into $L^{2}(\mathbb{R})$. Since $v^{*}(x) \rightarrow v^{\infty}$ for $|x| \rightarrow \infty$, the operator $\mathcal{G}$ is a relatively compact perturbation of $\mathcal{G}_{\infty}=\partial_{x}^{2}+H^{\prime}\left(v_{\infty}\right)$. By ODE-Hyperbolicity (2.4), we have $H^{\prime}\left(v_{\infty}\right)<0$ so that $\mathcal{G}_{\infty}$ is invertible and $\mathcal{G}$ is Fredholm with index 0 . Note that $v_{x}^{*}$ belongs to the kernel and is odd. By ODE-uniqueness (up to scalar multiples) of bounded solutions at $x=+\infty$, the kernel is at most one-dimensional, and therefore $\mathcal{G}$ is invertible on $H_{\text {even }}^{2}(\mathbb{R})$. Using the Implicit Function Theorem, we therefore find a local smooth solution $w(\cdot, \mu) \in H_{\text {even }}^{2}(\mathbb{R})$. One readily concludes that $w(x ; \mu) \rightarrow 0$ for $x \rightarrow \infty$, which gives the asymptotics of the spike solution $v=w+v^{\infty}$ as claimed.

\section{Linear and nonlinear instability}

In this section, we make precise the basic ingredient to our instability result, which is a linear instability result. Consider therefore the formal linearization of (1.1) along the spike $\left(u^{*}, v^{*}\right)$

$$
\frac{\mathrm{d}}{\mathrm{d} t}\left(\begin{array}{l}
u \\
v
\end{array}\right)=\mathcal{L}\left(\begin{array}{l}
u \\
v
\end{array}\right)
$$

where

$$
\mathcal{L}=\left[\begin{array}{cc}
\partial_{x}\left(a^{*} \partial_{x}+a_{u}^{*} u_{x}^{*}+b_{u}^{*} v_{x}^{*}\right) & \partial_{x}\left(b^{*} \partial_{x}+a_{v}^{*} u_{x}^{*}+b_{v}^{*} v_{x}^{*}\right) \\
f_{u}^{*} & \partial_{x}^{2}+f_{v}^{*}
\end{array}\right]
$$


We recall that here "*" next to any of the the functions $a, b, f$ and their partial derivatives represents the composition of the respective function with the spike $\left(u^{*}, v^{*}\right)$.

We can view the differential expression $\mathcal{L}$ as a densely defined, closed operator on $B U C\left(\mathbb{R}, \mathbb{C}^{2}\right)$ with domain $B U C^{2}\left(\mathbb{R}, \mathbb{C}^{2}\right)$, the functions with two bounded, uniformly continuous derivatives. In this case it actually is the linearization of the evolution to (1.1) at the equilibrium $\left(u^{*}, v^{*}\right)$.

It will however be convenient to also consider $\mathcal{L}$ as an operator on $L^{2}\left(\mathbb{R}, \mathbb{C}^{2}\right)$ with domain $H^{2}\left(\mathbb{R}, \mathbb{C}^{2}\right)$, and on certain weighted $L^{2}$-spaces. Since $\mathcal{L}$ is a lower-order perturbation of a diffusion operator, it generates an analytic semigroup with maximal regularity properties as described in [15], say.

Proposition 3.1. Under the assumptions of Theorem 1.1, the semigroup generated by $\mathcal{L}$ is unstable on $B U C\left(\mathbb{R}, \mathbb{C}^{2}\right)$. More precisely, the spectral radius of the semigroup in $B U C\left(\mathbb{R}, \mathbb{C}^{2}\right)$ is larger than one,

$$
r\left(\mathrm{e}^{\mathcal{L} t}\right)>1
$$

We will prove Proposition 3.1 in the next sections. Let us now briefly explain how Proposition 3.1 implies Theorem 1.1. We use a result of Henry [6, Thm 5.1.5], which states that an equilibrium to a discrete-time dynamical system in a Banach space is unstable if the spectral radius of the time-one map is larger than one. Well-posedness of the evolution equation [15] implies that the derivative of the time-one map at the equilibrium is given by $\mathrm{e}^{\mathcal{L}}$, which by Proposition 3.1 possesses a spectral radius larger than one. This proves Theorem 1.1.

\section{Essential spectrum}

In this section, we show that the essential spectrum of the linearization is unstable if the asymptotic equilibrium is unstable, $f_{v}\left(u^{\infty}, v^{\infty}\right)>0$. We also characterize the essential spectrum when $f_{v}\left(u^{\infty}, v^{\infty}\right)<0$.

We define the limiting operator $\mathcal{L}_{\infty}$ through

$$
\mathcal{L}_{\infty}=\left[\begin{array}{cc}
a^{\infty} \partial_{x}^{2} & b^{\infty} \partial_{x}^{2} \\
f_{u}^{\infty} & \partial_{x}^{2}+f_{v}^{\infty}
\end{array}\right] .
$$

Again, $\mathcal{L}_{\infty}$ can be considered on various function spaces, or merely as a differential expression, slightly abusing notation.

For a given choice of function space, we say $\lambda$ is in the essential spectrum $\sigma_{\text {ess }}(\mathcal{L})$ if $\mathcal{L}-\lambda$ is not Fredholm index zero. We refer to the complement of the essential spectrum in the spectrum as the point spectrum $\sigma_{\text {point }}(\mathcal{L})$.

Proposition 4.1. The essential spectra of the operators $\mathcal{L}$ and $\mathcal{L}_{\infty}$ coincide, and are equal for the choices of function space $X=L^{2}\left(\mathbb{R}, \mathbb{C}^{2}\right)$ and $X=B U C\left(\mathbb{R}, \mathbb{C}^{2}\right)$. Moreover, point spectrum of $\mathcal{L}$ and multiplicities are independent of the choice of function space, $X=L^{2}\left(\mathbb{R}, \mathbb{C}^{2}\right)$ and $X=B U C\left(\mathbb{R}, \mathbb{C}^{2}\right)$. 
Proof. Arguments similar to the ones given in [6, $\S 5$, Appendix] show that essential spectra are independent of the function spaces considered here. Also, eigenfunctions and generalized eigenfunctions to eigenvalues in the point spectrum decay exponentially at $\pm \infty$, so that point spectrum and multiplicities are independent of the function space. We need to show that essential spectra of $\mathcal{L}$ and $\mathcal{L}_{\infty}$ coincide. We therefore first notice that

$$
\tilde{\mathcal{L}}_{\infty}=\left[\begin{array}{cc}
a^{*} \partial_{x}^{2} & b^{*} \partial_{x}^{2} \\
f_{u}^{\infty} & \partial_{x}^{2}+f_{v}^{\infty}
\end{array}\right]
$$

is a compact perturbation of $\mathcal{L}$, so that essential spectra of $\tilde{\mathcal{L}}_{\infty}$ and $\mathcal{L}$ coincide. In order to see that the $x$-dependence in the highest-order derivatives does not alter the essential spectrum, we factor the eigenvalue problem as follows. Define

$$
D_{\infty}=\left[\begin{array}{cc}
a^{\infty} & b^{\infty} \\
0 & 1
\end{array}\right], D(x)=\left[\begin{array}{cc}
a^{*}(x) & b^{*}(x) \\
0 & 1
\end{array}\right], N_{\infty}=\left[\begin{array}{cc}
0 & 0 \\
f_{u}^{\infty} & f_{v}^{\infty}
\end{array}\right] .
$$

We can then factor the eigenvalue operator

$$
\tilde{\mathcal{L}}_{\infty}-\lambda=D(x) D_{\infty}^{-1}\left(D_{\infty} \partial_{x}^{2}+D_{\infty} D(x)^{-1}\left(N_{\infty}-\lambda\right)\right)
$$

Since $D(x)$ is invertible, Fredholm properties of $\tilde{\mathcal{L}}_{\infty}-\lambda$ and $D_{\infty} \partial_{x}^{2}+D_{\infty} D(x)^{-1}\left(N_{\infty}-\lambda\right)$ coincide. The latter is readily seen to be a compact perturbation of $\mathcal{L}_{\infty}-\lambda$, which shows that essential spectra of $\mathcal{L}_{\infty}$ and $\mathcal{L}$ coincide.

Now, the essential spectrum of the operator $\mathcal{L}$ can be computed readily via Fourier transform on $L^{2}\left(\mathbb{R}, \mathbb{C}^{2}\right)$ :

Lemma 4.2. The essential spectrum of $\mathcal{L}$ is given by

$$
\sigma_{\mathrm{ess}}(\mathcal{L})=\left\{\lambda_{ \pm}(\xi): \xi \in \mathbb{R}\right\}, \quad \text { where } \quad \lambda_{ \pm}(\xi)=\frac{\operatorname{tr}(\xi) \pm \sqrt{\operatorname{tr}(\xi)^{2}-4 \operatorname{det}(\xi)}}{2}
$$

with

$$
\operatorname{tr}(\xi)=-\left(a^{\infty}+1\right) \xi^{2}+f_{v}^{\infty}, \quad \operatorname{det}(\xi)=a^{\infty} \xi^{4}+\left(f_{u}^{\infty} b^{\infty}-f_{v}^{\infty} a^{\infty}\right) \xi^{2}
$$

Proof. Taking Fourier transform, we see that $\mathcal{L}_{\infty}$ is similar to the operator of multiplication by the matrix-valued function defined by $\hat{\mathcal{L}}_{\infty}(\xi)=-D_{\infty} \xi^{2}+N_{\infty}$, proving the lemma.

Remark 4.3. The lemma has some immediate consequences on stability of the essential spectrum.

(i) Note that $0 \in \sigma_{\text {ess }}(\mathcal{L})$ since $\operatorname{det}(0)=0$.

(ii) From ODE-Hyperbolicity (2.4), we conclude that $\operatorname{det}(\xi) \geq 0$. Therefore, $\sup \operatorname{Re} \sigma_{\text {ess }}(\mathcal{L}) \leq$ 0 if and only if $\operatorname{tr}(\xi) \leq 0$ for all $\xi$, which amounts to $f_{v}^{\infty} \leq 0$.

Lemma 4.4. Under the assumptions of Theorem 1.1, we have that $\operatorname{tr}(0)=f_{v}^{\infty} \neq 0$. 
(i) The essential spectral radius of $\mathrm{e}^{\mathcal{L}}$ is larger than 1 if $f_{v}^{\infty}>0$.

(ii) The essential spectral radius of $\mathrm{e}^{\mathcal{L}}$ is 1 if $f_{v}^{\infty}<0$. In fact, the semigroup generated by $\mathcal{L}_{\infty}$ on $L^{2}\left(\mathbb{R}, \mathbb{C}^{2}\right)$ is bounded in this case.

Proof. For spectral considerations, it is enough to consider $\mathcal{L}_{\infty}$ on $L^{2}\left(\mathbb{R}, \mathbb{C}^{2}\right)$ by Proposition 4.1. Lemma 4.2 and the spectral mapping for analytic semigroups then readily implies the statements on spectral radii. Boundedness of the semigroup in $L^{2}\left(\mathbb{R}, \mathbb{C}^{2}\right)$ readily follows from the representation of the semigroup in Fourier space.

Remark 4.5. When $f_{v}^{\infty}=0$, we have a Jordan block at $\xi=0$ in Fourier space, so that spatially homogeneous profiles grow linearly in time under the linear evolution. This shows linear instability in $B U C\left(\mathbb{R}, \mathbb{C}^{2}\right)$, and, using the Fourier multiplication representation, also in $L^{2}\left(\mathbb{R}, \mathbb{R}^{2}\right)$, in the marginal case $f_{v}^{\infty}=0$. Note that this linear instability need not imply nonlinear instability, as the example $u_{t}=0, v_{t}=u-v^{3}$ readily confirms. Figure 1 shows spectra when $\operatorname{det}(\xi) \geq 0$, for $f_{v}^{\infty}<0$ and $f_{v}^{\infty}>0$.

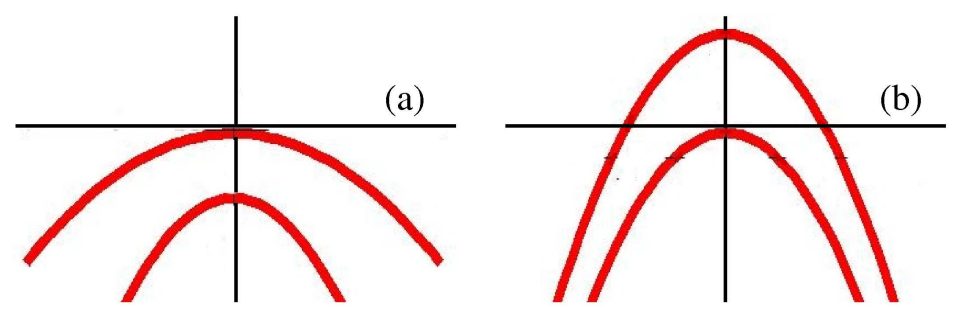

Figure 1: Schematic plots of the graphs of $\lambda_{ \pm}$in a neighborhood of $\xi=0$ are shown when (a) $f_{v}^{\infty}<0$ and (b) $f_{v}^{\infty}>0$.

\section{$5 \quad$ Tracing the point spectrum}

In the previous section, we concluded that there is essential spectrum with positive real part whenever $f_{v}^{\infty}>0$. In order to prove Proposition 4.1, it therefore is sufficient (and necessary) to show that there exists an eigenvalue in the point spectrum with positive real part whenever $f_{v}^{\infty}<0$, which will be the main result in this section.

The proof proceeds in several steps. We first construct a homotopy to a lower-triangular system, Section 5.1. During the homotopy, we control the existence of the nonlinear spike, and the structure of essential spectra. The next steps are to calculate the point spectrum at the end of the homotopy, Section 5.2, spectral properties at $\lambda=0$, Section $5.3-5.4$, asymptotics of eigenfunctions at $\lambda \sim 0$, Section 5.5, and finally calculate eigenvalues for $\lambda \sim 0$, Section 5.6. We conclude with the proof of Proposition 4.1 in Section 5.7. 


\subsection{Homotopy}

Our main goal in this subsection is to construct a homotopy to a "simpler" system, for which we can easily compute the point spectrum, without modifying the structure of the spikes and without changing the stability of $\sigma_{\text {ess }}(\mathcal{L})$. We introduce the homotopy parameter $\tau \in[0,1]$ and consider the system

$$
\left\{\begin{array}{l}
u_{t}=\left[a_{\tau}(u, v) u_{x}+b_{\tau}(u, v) v_{x}\right]_{x} \quad t \geq 0, x \in \mathbb{R} . \\
v_{t}=v_{x x}+\tilde{f}(u, v, \tau),
\end{array}\right.
$$

The functions $a_{\tau}, b_{\tau}: \mathbb{R}^{2} \rightarrow \mathbb{R}$ and $\tilde{f}: \mathbb{R}^{2} \times[0,1] \rightarrow \mathbb{R}$ are defined by

$$
\begin{aligned}
a_{\tau}(u, v) & =(1-\tau) a(u, v)+\tau, \quad b_{\tau}(u, v)=(1-\tau) b(u, v), \\
\tilde{f}(u, v, \tau) & =f(u, v)-f\left(\varphi_{\tau}(v), v\right)+f\left(\varphi_{0}(v), v\right),
\end{aligned}
$$

where $\varphi_{\tau}$ is the solution of the Cauchy problem

$$
\frac{d u}{d v}=-\frac{b_{\tau}(u, v)}{a_{\tau}(u, v)}, \quad u\left(v^{\infty}\right)=u^{\infty} .
$$

Remark 5.1. We collect a few properties of this particular homotopy.

(i) The homotopy originates at our equation (1.1), $\tilde{f}(u, v, 0)=f(u, v)$;

(ii) If we define $u_{\tau}^{*}:=\varphi_{\tau}\left(v^{*}\right)$ and $v_{\tau}^{*}:=v^{*}$ then $\left(u_{\tau}^{*}, v_{\tau}^{*}\right)$ is a spike for (5.1);

(iii) The background states for the system (5.1), $\lim _{x \rightarrow \pm \infty} u_{\tau}^{*}(x)=u^{\infty}$ and $\lim _{x \rightarrow \pm \infty} v_{\tau}^{*}(x)=$ $v^{\infty}$, do not depend on $\tau$.

The linearization of (5.1) along the spike $\left(u_{\tau}^{*}, v_{\tau}^{*}\right)$ on $X \times X$, where $X$ is a function space, is given by

$$
\frac{\mathrm{d}}{\mathrm{d} t}\left(\begin{array}{l}
u \\
v
\end{array}\right)=\mathcal{L}_{\tau}\left(\begin{array}{l}
u \\
v
\end{array}\right)
$$

where $\mathcal{L}_{\tau}$ is defined by replacing $a, b$ and $f$ in the definition $(3.2)$ of $\mathcal{L}$ by $a_{\tau}, b_{\tau}$ and $\tilde{f}(\cdot, \cdot, \tau)$, respectively.

Lemma 5.2. If $f_{v}^{\infty}<0$ the essential spectrum of $\mathcal{L}_{\tau}$ is stable.

Proof. From Lemma 4.4 it follows that it suffices to show that $\tilde{f}_{v}^{\infty}<0$. We calculate

$$
\begin{aligned}
\tilde{f}_{v}(u, v, \tau)= & f_{v}(u, v)-f_{u}\left(\varphi_{\tau}(v), v\right) \varphi_{\tau}^{\prime}(v)-f_{v}\left(\varphi_{\tau}(v), v\right) \\
& +f_{u}\left(\varphi_{0}(v), v\right) \varphi_{0}^{\prime}(v)+f_{v}\left(\varphi_{0}(v), v\right) .
\end{aligned}
$$

Using Remark 5.1(iii), we obtain

$$
\tilde{f}_{v}^{\infty}=f_{v}^{\infty}+f_{u}^{\infty}\left(\frac{b_{\tau}^{\infty}}{a_{\tau}^{\infty}}-\frac{b^{\infty}}{a^{\infty}}\right)=f_{v}^{\infty}-\frac{b^{\infty}}{a^{\infty}} f_{u}^{\infty} \frac{\tau}{\tau+(1-\tau) a^{\infty}}:=p(\tau) .
$$

Since $p$ is a monotone function on $[0,1]$ and $p(0)=f_{v}^{\infty}<0$ and $p(1)=f_{v}^{\infty}-\frac{b^{\infty}}{a^{\infty}} f_{u}^{\infty}<0$, by ODE-Hyperbolicity, (2.4), we conclude that $\tilde{f}_{v}^{\infty}<0$, proving the lemma. 


\subsection{Instability at $\tau=1$}

At the end of the homotopy, the system possesses a lower triangular structure and we can readily infer instability.

Lemma 5.3. If $f_{v}^{\infty}<0$, then the spikes in the system (5.1) with $\tau=1$ are unstable. Moreover, $\mathcal{L}_{1}$ has precisely one positive eigenvalue.

Proof. We note that if $\tau=1$ the operator $\mathcal{L}_{1}$ has lower triangular block structure

$$
\mathcal{L}_{1}=\left[\begin{array}{cc}
\partial_{x}^{2} & 0 \\
* & \mathcal{K}
\end{array}\right],
$$

where $\mathcal{K}=\partial_{x}^{2}+H_{1}^{\prime}\left(v^{*}\right)$ and $H_{1}(v)=\tilde{f}\left(\varphi_{1}(v), v, 1\right)$. Therefore, the spectrum of $\mathcal{L}_{1}$ is the union of the spectra of $\partial_{x}^{2}$ and $\mathcal{K}$. We now proceed as in the proof of Lemma 2.1. Since $\mathcal{K}$ is Sturm-Liouville, its first eigenfunction is positive. Since $v_{x}^{*}$ belongs to the kernel of $\mathcal{K}$ and has precisely one sign change, we infer that $\mathcal{K}$, and hence $\mathcal{L}$, has precisely one unstable eigenvalue.

The remainder of the proof aims at conserving this unstable real eigenvalue. We will therefore study in detail properties of the operators $\mathcal{L}_{\tau}-\lambda$ at and near $\lambda=0$ in order to prevent the unstable eigenvalue from crossing the origin. In the sequel, when we study the operator $\mathcal{L}_{\tau}$, we simply write $\mathcal{L}$ for ease of notation, since operators do not change qualitatively during the homotopy.

\subsection{The kernel of $\mathcal{L}$}

From our assumptions on $a, b, f$, one can see that the kernel of $\mathcal{L}$ (and of $\mathcal{L}_{\tau}$ for all $\tau$ ) consists of smooth functions for all functions spaces in consideration here. In fact, functions in the kernel solve the system of ODEs

$$
\begin{gathered}
a^{*} u_{x}+\left(a_{u}^{*} u_{x}^{*}+b_{u}^{*} v_{x}^{*}\right) u+b^{*} v_{x}+\left(a_{v}^{*} u_{x}^{*}+b_{v}^{*} v_{x}^{*}\right) v=0 \\
v_{x x}+f_{u}^{*} u+f_{v}^{*} v=0 .
\end{gathered}
$$

If we require boundedness of $u$ and $v$, we can solve the first equation (5.4) explicitly.

Lemma 5.4. If $u, v \in B U C(\mathbb{R})$ satisfy equation (5.4) then $u=\alpha\left(\partial_{\mu} u_{\mid \mu=0}^{*}+\frac{b^{*}}{a^{*}} \partial_{\mu} v_{\mid \mu=0}^{*}\right)-\frac{b^{*}}{a^{*}} v$, for some constant $\alpha \in \mathbb{C}$. Here, $\left(u^{*}(\cdot, \mu), v^{*}(\cdot, \mu)\right)$ refers to the family of spikes as constructed in Lemma 2.1.

Proof. One readily verifies that for any fixed $v$, a solution to equation (5.4) is given by $u=-\frac{b^{*}}{a^{*}} v$. Of course, this relation can also be obtained by linearizing the nonlinear flow solution (2.2) of (1.4) with respect to $v$.

Since (5.4) can be considered as an inhomogeneous linear equation

$$
a^{*} u_{x}+\left(a_{u}^{*} u_{x}^{*}+b_{u}^{*} v_{x}^{*}\right) u=-\left(b^{*} v_{x}+a_{v}^{*} u_{x}^{*} v+b_{v}^{*} v_{x}^{*} v\right),
$$


the general solution of (5.4) has the form

$$
u=\alpha z-\frac{b^{*}}{a^{*}} v
$$

for some constant $\alpha \in \mathbb{C}$, where $z$ is a solution of the equation

$$
a^{*} z_{x}+\left(a_{u}^{*} u_{x}^{*}+b_{u}^{*} v_{x}^{*}\right) z=0
$$

Using the fact that $\left(u^{*}(\cdot, \mu), v^{*}(\cdot, \mu)\right)$ is a family of spikes we obtain that

$$
a\left(u^{*}(x, \mu), v^{*}(x, \mu)\right) u_{x}^{*}(x, \mu)+b\left(u^{*}(x, \mu), v^{*}(x, \mu)\right) v_{x}^{*}(x, \mu)=0 .
$$

Differentiating this equation with respect to $\mu$ and then setting $\mu=0$, we find the general solution $z=\partial_{\mu} u_{\mid \mu=0}^{*}+\frac{b^{*}}{a^{*}} \partial_{\mu} v_{\mid \mu=0}^{*}$, which proves the lemma.

Lemma 5.5. A pair $(u, v)$ belongs to the kernel of $\mathcal{L}$ in $B U C\left(\mathbb{R}, \mathbb{C}^{2}\right)$ if and only if for some $\alpha, \beta \in \mathbb{C}$,

$$
u=\alpha \partial_{\mu} u_{\mid \mu=0}^{*}+\beta u_{x}^{*} \quad v=\alpha \partial_{\mu} v_{\mid \mu=0}^{*}+\beta v_{x}^{*} .
$$

Proof. From Lemma 5.4 we know that $u=\alpha\left(\partial_{\mu} u_{\mid \mu=0}^{*}+\frac{b^{*}}{a^{*}} \partial_{\mu} v_{\mid \mu=0}^{*}\right)-\frac{b^{*}}{a^{*}} v$, for some constant $\alpha \in \mathbb{C}$. Substituting this expression into equation (5.5) we obtain that a function $v$ from the kernel of $\mathcal{L}$ in $B U C$ satisfies the following equation

$$
v_{x x}+\left(f_{v}^{*}-f_{u}^{*} \frac{b^{*}}{a^{*}}\right) v=-\alpha\left(f_{u}^{*} \partial_{\mu} u_{\mid \mu=0}^{*}+\frac{b^{*}}{a^{*}} \partial_{\mu} v_{\mid \mu=0}^{*}\right) .
$$

Once again using the fact that $\left(u^{*}(\cdot, \mu), v^{*}(\cdot, \mu)\right)$ is a family of spikes, that $v^{*}(\cdot, \mu)$ satisfies the equation

$$
v_{x x}^{*}(x, \mu)+f\left(u^{*}(x, \mu), v^{*}(x, \mu)\right)=0 .
$$

Differentiating with respect to $\mu$ in this equation and setting $\mu=0$, we infer that $\alpha \partial_{\mu} v_{\mid \mu=0}^{*}$ is a particular solution of equation (5.7). Hence, the general solution of equation (5.7) is of the form $v=\alpha \partial_{\mu} v_{\mid \mu=0}^{*}+\tilde{v}$, where $\tilde{v}$ is a solution of the equation

$$
\tilde{v}_{x x}+\left(f_{v}^{*}-f_{u}^{*} \frac{b^{*}}{a^{*}}\right) \tilde{v}=0 .
$$

Since equation (5.8) is the variational equation of $v_{x x}+H(v)=0$, with $H(v)=f\left(\varphi_{0}(v), v\right)$, defined in (2.3), we infer that $\tilde{v}=\beta v_{x}^{*}$ for some constant $\beta \in \mathbb{C}$.

Corollary 5.6. We have that

(i) $\operatorname{ker} \mathcal{L}=\operatorname{Span}\left\{\left(\partial_{\mu} u_{\mid \mu=0}^{*}, \partial_{\mu} v_{\mid \mu=0}^{*}\right)^{\mathrm{T}}\right\}$, in $B U C_{\text {even }}$;

(ii) $\operatorname{ker} \mathcal{L}=\operatorname{Span}\left\{\left(u_{x}^{*}, v_{x}^{*}\right)^{\mathrm{T}}\right\}$, in $B U C_{\text {odd }}$.

That is, the kernel is one-dimensional in spaces of even and odd functions. Moreover, the kernel in $B U C_{\text {even }}$ is spanned by a function which converges to $\partial_{\mu}\left(u^{\infty}(0), v^{\infty}(0)\right)^{\mathrm{T}}$. By Lemma 2.1, this limit does not vanish under the assumptions of Theorem 1.1. 


\subsection{The Fredholm properties of $\mathcal{L}$ on weighted spaces}

In this section we prove that $\mathcal{L}$ (and $\mathcal{L}_{\tau}$ ) is Fredholm on exponentially weighted spaces. Therefore, define the space $L_{\eta}^{2}\left(\mathbb{R}, \mathbb{C}^{2}\right)$ via the weighted $L^{2}$-norm

$$
\|u\|_{\eta}^{2}=\int_{\mathbb{R}}\left|u(x) \mathrm{e}^{\eta|x|}\right|^{2} \mathrm{~d} x
$$

for $\eta \in \mathbb{R}$, and its even and odd subspaces $L_{\eta, \text { even }}^{2}\left(\mathbb{R}, \mathbb{C}^{2}\right)$ and $L_{\eta, \text { odd }}^{2}\left(\mathbb{R}, \mathbb{C}^{2}\right)$. More generally, we can define the weighted $L^{2}$-spaces $L^{2}\left(\mathbb{R}, \mathbb{C}^{2}, \omega \mathrm{d} x\right)$ with weight function $\omega(x)$ and norm

$$
\|u\|_{\omega}^{2}=\int_{\mathbb{R}}|u(x)|^{2} \omega(x) \mathrm{d} x .
$$

The following lemma shows that $\mathcal{L}$ is Fredholm on $L_{\eta}^{2}\left(\mathbb{R}, \mathbb{C}^{2}\right), \eta>0$ small.

Lemma 5.7. There exists $\eta^{*}>0$ such that for all $\eta \in\left(0, \eta^{*}\right), \mathcal{L}$ is Fredholm on $L_{\eta}^{2}\left(\mathbb{R}, \mathbb{C}^{2}\right)$ with index $\operatorname{ind}(\mathcal{L})=-2$.

Proof. The strategy of the proof is as follows. We first show that $\mathcal{L}$ on the weighted space is conjugate to an operator on $L^{2}$ without weights. We then rewrite this operator as a firstorder differential operator on $L^{2}\left(\mathbb{R}, \mathbb{C}^{4}\right)$. The Fredholm index of this first-order operator can be computed from Morse indices of its limiting matrices, using a result of Palmer [16, 17].

We start by introducing a smoothed weight function $\psi \in C^{\infty}(\mathbb{R})$, which satisfies $\psi(x)=$ $e^{-\eta|x|}$ for all $x \in \mathbb{R}$ with $|x| \geq 1$ and $\inf _{|x| \leq 1} \psi(x)>0$. We then find that $L_{\eta}^{2}\left(\mathbb{R}, \mathbb{C}^{2}\right)=$ $L^{2}\left(\mathbb{R}, \mathbb{C}^{2},[\psi(x)]^{-2} d x\right)$ with equivalent norms $\|\cdot\|_{L_{\eta}^{2}}$ and $\|\cdot\|_{L_{\psi^{-2}}^{2}} \cdot$

Next, define the isomorphism $U_{\psi}: L^{2}\left(\mathbb{R}, \mathbb{C}^{2}\right) \rightarrow L_{\eta}^{2}(\mathbb{R}, \mathbb{C})$ by $U_{\psi} w=\psi w$. One readily verifies that $U_{\psi}$ is a bounded, invertible operator with bounded inverse. In consequence, the operator $\mathcal{L}$ is Fredholm on $L_{\eta}^{2}\left(\mathbb{R}, \mathbb{C}^{2}\right)$ if and only if $\mathcal{L}_{\psi}=U_{\psi}^{-1} \mathcal{L} U_{\psi}$ is Fredholm on $L^{2}\left(\mathbb{R}, \mathbb{C}^{2}\right)$ and their indices coincide.

Now, recall that we can write $\mathcal{L}=D(x) \partial_{x}^{2}+M(x) \partial_{x}+N(x)$, where the matrix-valued functions $D, M$ and $N$ are continuous, $D(x)$ is invertible for all $x \in \mathbb{R}$ and $D(x) \rightarrow D_{\infty}, M(x) \rightarrow 0$ and $N(x) \rightarrow N_{\infty}$, for $|x| \rightarrow \infty$. Here,

$$
D_{\infty}=\left[\begin{array}{cc}
a^{\infty} & b^{\infty} \\
0 & 1
\end{array}\right] \quad \text { and } \quad N_{\infty}=\left[\begin{array}{cc}
0 & 0 \\
f_{u}^{\infty} & f_{v}^{\infty}
\end{array}\right]
$$

It follows that

$$
\mathcal{L}_{\psi}=D(x)\left(\partial_{x}+\frac{\psi^{\prime}(x)}{\psi(x)}\right)^{2}+M(x)\left(\partial_{x}+\frac{\psi^{\prime}(x)}{\psi(x)}\right)+N(x) .
$$

Next, we define the matrix-valued functions $A, A_{\psi}: \mathbb{R} \rightarrow \mathbb{C}^{4 \times 4}$ by

$$
\begin{gathered}
A(x)=\left[\begin{array}{cc}
0_{2} & I_{2} \\
-D(x)^{-1} N(x) & -D(x)^{-1} M(x)
\end{array}\right] \\
A_{\psi}(x)=\left[\begin{array}{cc}
0_{2} & I_{2} \\
-D(x)^{-1} N(x)-\frac{\psi^{\prime}(x)}{\psi(x)} D(x)^{-1} M(x)-\frac{\psi^{\prime \prime}(x)}{\psi(x)} & -D(x)^{-1} M(x)+2 \frac{\psi^{\prime}(x)}{\psi(x)}
\end{array}\right],
\end{gathered}
$$


where $0_{2}=\left[\begin{array}{ll}0 & 0 \\ 0 & 0\end{array}\right]$ and $I_{2}=\left[\begin{array}{ll}1 & 0 \\ 0 & 1\end{array}\right]$. From [19, Thm. A1] it follows that $\mathcal{L}_{\psi}$ is Fredholm on $L^{2}\left(\mathbb{R}, \mathbb{C}^{2}\right)$ if and only if $\mathcal{T}_{\psi}=\partial_{x}-A_{\psi}(x)$ is Fredholm on $L^{2}\left(\mathbb{R}, \mathbb{C}^{4}\right)$ and their indices coincide. Let $J_{\psi}: \mathbb{R} \rightarrow \mathbb{C}^{4 \times 4}$ be the matrix-valued function defined by

$$
J_{\psi}(x)=\left[\begin{array}{cc}
I_{2} & \left(1-\frac{\psi^{\prime}(x)}{\psi(x)}\right) I_{2} \\
0_{2} & I_{2}
\end{array}\right] .
$$

Since $J_{\psi}$ is continuous on $\mathbb{R}$ and $\operatorname{det} J_{\psi}(x)=1$ for all $x \in \mathbb{R}$, the operator of multiplication by $J_{\psi}$ is bounded, invertible with bounded inverse on $L^{2}\left(\mathbb{R}, \mathbb{C}^{4}\right)$. Using this transformation, one can see that $\mathcal{T}_{\psi}$ is conjugate to $\tilde{\mathcal{T}}_{\psi}=\partial_{x}+\frac{\psi^{\prime}(x)}{\psi(x)} I_{4}-A(x)$. Hence, $\mathcal{L}$ is Fredholm on $L_{\eta}^{2}\left(\mathbb{R}, \mathbb{C}^{2}\right)$ if and only if $\tilde{\mathcal{T}}_{\psi}$ is Fredholm on $L^{2}\left(\mathbb{R}, \mathbb{C}^{4}\right)$.

We now investigate the limiting behavior of the coefficients of $\tilde{\mathcal{T}}_{\psi}$. First note that

$$
A(x) \underset{|x| \rightarrow \infty}{\longrightarrow} A_{\infty}, \quad \text { where } \quad A_{\infty}=\left[\begin{array}{cc}
0_{2} & I_{2} \\
-D_{\infty}^{-1} N_{\infty} & 0_{2}
\end{array}\right]
$$

Palmer's classical result $([16,17])$ states that the operator $\tilde{\mathcal{T}}_{\psi}$ is Fredholm if and only if $A_{ \pm, \eta}:=$ $\lim _{x \rightarrow \pm \infty}\left(A(x)-\frac{\psi^{\prime}(x)}{\psi(x)} I_{4}\right)$ are hyperbolic, and its Fredholm index is given by the difference of the Morse indices,

$$
\operatorname{ind}\left(\tilde{\mathcal{T}}_{\psi}\right)=i\left(A_{-, \eta}\right)-i\left(A_{+\eta}\right)
$$

In our case, the eigenvalues of $A_{\infty}$ are $\pm \sqrt{\frac{b^{\infty}}{a^{\infty}} f_{u}^{\infty}-f_{v}^{\infty}}$ with multiplicity 1 and 0 with multiplicity 2. Since $\lim _{x \rightarrow \pm \infty} \frac{\psi^{\prime}(x)}{\psi(x)}=\mp \eta$ we obtain that $A_{ \pm, \eta}=A_{\infty} \pm \eta I_{4}$. Taking $\eta^{*}=\frac{1}{2} \sqrt{\frac{b^{\infty}}{a^{\infty}} f_{u}^{\infty}-f_{v}^{\infty}}$ we have that $A_{ \pm, \eta}$ are hyperbolic, and the dimension of the unstable subspaces are the Morse indices $i\left(A_{-, \eta}\right)=1$ and $i\left(A_{+\eta}\right)=3$. This shows that $\tilde{\mathcal{T}}_{\psi}$ is Fredholm with index $\operatorname{ind}\left(\tilde{\mathcal{T}}_{\psi}\right)=-2$, and proves the lemma.

Lemma 5.8. For all $\eta \in\left(0, \eta^{*}\right)$ the operator $\mathcal{L}$ is Fredholm with index -1 on $L_{\eta, \text { even/odd }}^{2}\left(\mathbb{R}, \mathbb{C}^{2}\right)$.

Proof. Define the operators $\mathcal{L}_{\text {e/o }}$ as the restriction of $\mathcal{L}$ on $H_{\eta, \text { even/odd }}^{2}\left(\mathbb{R}, \mathbb{C}^{2}\right)$. Since $\mathcal{L}$ leaves invariant the space of even or odd functions, we have

$$
\operatorname{ind}\left(\mathcal{L}_{\mathrm{e}}\right)+\operatorname{ind}\left(\mathcal{L}_{\mathrm{o}}\right)=\operatorname{ind}(\mathcal{L})=-2
$$

Next, we denote by

$$
Z_{\eta, \text { odd }}=\left\{z \in H_{\eta, \text { odd }}^{2}\left(\mathbb{R}, \mathbb{C}^{2}\right): z^{\prime}(0)=0\right\} \quad \text { and } \quad \mathcal{L}_{Z}=\mathcal{L}_{\mid Z_{\eta, \text { odd }}}
$$

One readily verifies that $Z_{\eta \text {,odd }}$ is a closed subspace and $\operatorname{codim}\left(Z_{\eta \text {,odd }}\right)=2$. Thus, $\operatorname{ind}\left(\mathcal{L}_{Z}\right)=$ $\operatorname{ind}\left(\mathcal{L}_{\mathrm{o}}\right)-2$. Define the operators $\mathcal{P}: L_{\eta, \text { odd }}^{2}\left(\mathbb{R}, \mathbb{C}^{2}\right) \rightarrow L_{\eta, \text { even }}^{2}\left(\mathbb{R}, \mathbb{C}^{2}\right)$ by $(\mathcal{P} w)(x)=w(|x|)$ and $\mathcal{P}_{Z}: Z_{\eta, \text { odd }} \rightarrow H_{\eta, \text { even }}^{2}\left(\mathbb{R}, \mathbb{C}^{2}\right)$ by $\left(\mathcal{P}_{Z} z\right)(x)=z(|x|)$. We note that $\mathcal{P}$ is bounded, invertible, with bounded inverse and

$$
\operatorname{ker} \mathcal{P}_{Z}=\{0\} \quad \text { and } \quad \operatorname{im} \mathcal{P}_{Z}=\left\{w \in H_{\eta, \text { even }}^{2}\left(\mathbb{R}, \mathbb{C}^{2}\right): w(0)=0\right\}
$$


Thus, $\mathcal{P}$ and $\mathcal{P}_{Z}$ are Fredholm and $\operatorname{ind}(\mathcal{P})=0$ and $\operatorname{ind}\left(\mathcal{P}_{Z}\right)=-2$. Using the fact that the matrix-valued functions $D(\cdot)$ and $N(\cdot)$ are even and $M(\cdot)$ is odd, we infer $\mathcal{L}_{\mathrm{e}} \mathcal{P}_{Z}=\mathcal{P} \mathcal{L}_{Z}$. It follows that

$$
\begin{aligned}
\operatorname{ind}\left(\mathcal{L}_{\mathrm{o}}\right) & =\operatorname{ind}\left(\mathcal{L}_{Z}\right)+2=\operatorname{ind}\left(\mathcal{P} \mathcal{L}_{Z}\right)+2=\operatorname{ind}\left(\mathcal{L}_{\mathrm{e}} \mathcal{P}_{Z}\right)+2 \\
& =\operatorname{ind}\left(\mathcal{L}_{\mathrm{e}}\right)+\operatorname{ind}\left(\mathcal{P}_{Z}\right)+2=\operatorname{ind}\left(\mathcal{L}_{\mathrm{e}}\right) .
\end{aligned}
$$

Using (5.9), the lemma follows shortly.

We next briefly consider the adjoint $\mathcal{L}^{*}$ of $\mathcal{L}$. We compute the adjoint with respect to the non-weighted $L^{2}$-scalar product, so that $\mathcal{L}^{*}$ is a closed operator on $L_{-\eta}^{2}\left(\mathbb{R}, \mathbb{C}^{2}\right)$, given through

$$
\mathcal{L}^{*}=\left[\begin{array}{cc}
\left(a^{*} \partial_{x}-a_{u}^{*} u_{x}^{*}-b_{u}^{*} v_{x}^{*}\right) \partial_{x} & f_{u}^{*} \\
\left(b^{*} \partial_{x}-a_{v}^{*} u_{x}^{*}-b_{v}^{*} v_{x}^{*}\right) \partial_{x} & \partial_{x}^{2}+f_{v}^{*}
\end{array}\right] .
$$

Lemma 5.9. For all $\eta \in\left(0, \eta^{*}\right)$, $\operatorname{ker} \mathcal{L}^{*}$ in $L_{-\eta, \text { even }}^{2}\left(\mathbb{R}, \mathbb{C}^{2}\right)$ is spanned by $(1,0)^{\mathrm{T}}$.

Proof. From Corollary 5.6, the kernel of $\mathcal{L}$ in $B U C_{\text {even }}$ is one-dimensional, and limits of nontrivial functions in the kernel at $x= \pm \infty$ do not vanish. Therefore, the kernel of $\mathcal{L}$ in $L_{\eta, \text { even }}^{2}\left(\mathbb{R}, \mathbb{C}^{2}\right)$ is trivial. Since $\operatorname{ind}(\mathcal{L})=-1$ in $L_{\eta, \text { even }}^{2}\left(\mathbb{R}, \mathbb{C}^{2}\right)$ we infer from standard Fredholm theory that $\operatorname{ind}\left(\mathcal{L}^{*}\right)=1$. Also, the cokernel of $\mathcal{L}^{*}$ is trivial since the kernel of $\mathcal{L}$ is trivial. As a consequence, $\operatorname{dim} \operatorname{ker} \mathcal{L}^{*}=1$ in $L_{-\eta \text {,even }}^{2}(\mathbb{R}, \mathbb{C})$. The explicit expression (5.10) clearly admits the trivial kernel $(1,0)^{\mathrm{T}}$, which concludes the proof of the lemma.

\subsection{Asymptotics of eigenfunctions}

Next, we consider the eigenvalue problem associated with the operator $\mathcal{L}_{\infty}$,

$$
\left\{\begin{array}{l}
a^{\infty} u_{x x}+b^{\infty} v_{x x}=\lambda u \\
v_{x x}+f_{u}^{\infty} u+f_{v}^{\infty} v=\lambda v
\end{array}\right.
$$

We will see in the next section that exponential decay behavior of solutions to (5.11) correctly predicts exponential decay behavior of possible eigenfunctions of $\mathcal{L}$. In the sequel, we determine those asymptotic decay rates for (5.11). We therefore define the linear dispersion relation

$$
\Lambda(\lambda, \nu)=\left[\begin{array}{cc}
a^{\infty} \nu^{2}-\lambda & b^{\infty} \nu^{2} \\
f_{u}^{\infty} & \nu^{2}+f_{v}^{\infty}-\lambda
\end{array}\right] \quad \text { and } \quad d(\lambda, \nu)=\operatorname{det} \Lambda(\lambda, \nu) .
$$

Lemma 5.10. The solutions to (5.11) for $\lambda$ in a complex neighborhood of the origin can be characterized as follows.

(i) In case $\operatorname{Re} \lambda>0$, there are two roots of the equation $d(\lambda, \nu)=0$ with $\operatorname{Re} \nu_{j}>0, j=1,2$. The solutions bounded at $\pm \infty$, respectively, are of the form

$$
(u(x), v(x))^{\mathrm{T}}=C_{1} e^{\mp \nu_{1} x}+C_{2} e^{\mp \nu_{2} x} .
$$

Here, $C_{j}, j=1,2$ are vectors in the kernel of $\Lambda(\lambda, \nu)$ and $\nu_{j}, j=1,2$ are the roots of the equation $d(\lambda, \nu)=0$ with $\operatorname{Re} \nu_{j}>0, j=1,2$. 
(ii) Setting $\lambda=\gamma^{2}$, the $\nu_{j}, j=1,2$, can be considered as analytic functions of $\gamma$ with expansion

$$
\nu_{1}(\gamma)=\sqrt{\frac{f_{v}^{\infty}}{a^{\infty} f_{v}^{\infty}-b^{\infty} f_{u}^{\infty}}} \gamma+\mathcal{O}\left(\gamma^{2}\right) \quad \nu_{2}(\gamma)=\sqrt{-H^{\prime}\left(v^{\infty}\right)}+\mathcal{O}\left(\gamma^{2}\right),
$$

where $H$ was defined in (2.3), and $H^{\prime}<0$ is guaranteed by (2.4). Moreover, the constant $C_{1}$ can be chosen as an analytic function $C_{1}=\alpha(\gamma)$ with expansion

$$
\alpha(\gamma)=\left(-f_{v}^{\infty}, f_{u}^{\infty}\right)^{\mathrm{T}}+\mathrm{O}(\gamma)
$$

such that the function defined by $\alpha(\gamma) e^{ \pm \nu_{1}(\gamma) x}$ satisfies (5.11).

Proof. Solutions are clearly given by linear combinations of exponentials, and possibly algebraic factors, in case of double roots. Note that $\nu=\mathrm{i} k \in \mathrm{i} \mathbb{R}$ does not solve $d(\lambda, \nu)=0$ for $\operatorname{Re} \lambda>0$, since otherwise the essential spectrum would be unstable. Moreover, reflection symmetry, $\nu \mapsto-\nu$, or the fact that $\Lambda$ depends on $\nu^{2}$, only, shows that expansions at $\pm \infty$ are equivalent as stated. We will next compute the expansions for $\nu$ in terms of $\gamma=\sqrt{\lambda}$, which will also show that the roots $\nu_{j}$ are distinct and hence show that solutions are actually linear combinations of exponentials, only.

The equation $d(0, \nu)=0$ has 0 as a double root and $\pm \sqrt{-H^{\prime}\left(v^{\infty}\right)}$ as simple roots. Expanding the simple roots in $\gamma^{2}$ readily gives the expansions $\nu_{2}$. In order to derive an expansion for $\nu_{1}$, we expand $d\left(\gamma^{2}, \nu\right)$ in $\gamma^{2}$, to arrive at the analytic equation

$$
\nu^{2}\left(a^{\infty} f_{v}^{\infty}-b^{\infty} f_{u}^{\infty}\right)=f_{v}^{\infty} \gamma^{2}+\mathcal{O}\left(\left|\gamma^{4}\right|+|\nu|^{4}\right) .
$$

Newton's polygon readily gives the two unique solution branches $\pm \nu_{1}(\gamma)$.

Next, we substitute $\nu_{1}(\gamma)$ into the equation for eigenvectors near $\gamma=0$. Substituting the ansatz $\left(-f_{v}^{\infty}, f_{u}^{\infty}\right)^{\mathrm{T}}+\alpha_{2}(\gamma)$, we find an equations for $\alpha_{2}(\gamma)$ that we can easily solve to find the unique (up to scalar multiples) analytic eigenvector $\alpha(\gamma)$.

\subsection{The eigenvalue problem near 0 in $L_{\eta, \text { even }}^{2}\left(\mathbb{R}, \mathbb{C}^{2}\right)$}

We are now ready to address the eigenvalue problem for the linearization $\mathcal{L}$ near $\lambda=0$ using Lyapunov-Schmidt reduction. The following proposition states that the eigenvalue problem can be reduced to finding the roots of a single scalar function. Moreover, we are able to calculate the expansion of this scalar function near $\lambda=0$ in terms of the asymptotic states.

Proposition 5.11. Under the assumptions of Proposition 4.1, there exists $\delta>0$ and an analytic function $E: D(0, \delta) \rightarrow \mathbb{C}$, such that for any $\gamma>0$,

$$
\gamma^{2} \in \sigma_{\text {point }}(\mathcal{L}) \text { if and only if } E(\gamma)=0 .
$$

Moreover, we have the expansion

$$
E(\gamma)=-2 \sqrt{f_{v}^{\infty}\left(a^{\infty} f_{v}^{\infty}-b^{\infty} f_{u}^{\infty}\right)} \gamma+\mathcal{O}\left(\gamma^{2}\right)
$$

and $E^{\prime}(0) \neq 0$.

Proof. The proof is broken up into six steps. 
Step 1: The ansatz. We are interested in solutions to

$$
\left(\mathcal{L}-\gamma^{2}\right)\left(\begin{array}{l}
u \\
v
\end{array}\right)=0
$$

for $\gamma \sim 0$. We use the ansatz

$$
(u, v)^{\mathrm{T}}=w+\beta \alpha(\gamma)\left(\chi_{+} e^{-\nu_{1}(\gamma) \cdot}+\chi_{-} e^{\nu_{1}(\gamma) \cdot}\right)
$$

where $w \in L_{\eta \text {,even }}^{2}\left(\mathbb{R}, \mathbb{C}^{2}\right), \beta \in \mathbb{C}, \alpha(\cdot)$ and $\nu_{1}(\cdot)$ are defined in Lemma 5.10. Here $\chi_{+}(x)=$ $\frac{1-\rho(x)}{2}$, where $\rho \in C^{\infty}(\mathbb{R})$ is a smooth even function satisfying $\rho(x)=-1$ for all $x \leq-1$, $\rho(x)=1$ for all $x \geq 1$ and $\chi_{-}(x)=1-\chi_{+}(x)$. Clearly, a function $(u, v)^{\mathrm{T}}$ of the form (5.15) that solves the eigenvalue equation (5.14) for $\gamma>0$ belongs to the kernel of $\mathcal{L}$. We will see in Step 6 that any eigenfunction is actually of the form (5.15).

Summarizing, the ansatz allows us to consider the eigenvalue problem in the smaller space $L_{\eta, \text { even }}^{2}\left(\mathbb{R}, \mathbb{C}^{2}\right)$, only, instead of $L_{\text {even }}^{2}\left(\mathbb{R}, \mathbb{C}^{2}\right)$, at the expense of adding a free parameter $\beta$.

At $\gamma=\lambda=0$, there is a unique solution (up to scalar multiples) of the form (5.15), which solves (5.14), which is the kernel spanned by the tangent to the family of spikes, $\partial_{\mu}\left(u^{*}(\cdot, \mu), v^{*}(\cdot, \mu)\right)^{\mathrm{T}}$ at $\mu=0$. Indeed, all solutions of the form (5.15) at $\gamma=0$ are bounded, and, by Corollary 5.6 , the kernel on spaces of bounded functions is one-dimensional and spanned by this tangent vector to the family of spikes. Since spikes are exponentially localized, we find that

$$
\begin{aligned}
w_{0} & :=\left(\partial_{\mu} u_{\mid \mu=0}^{*}-\partial_{\mu} u^{\infty}(0), \partial_{\mu} v_{\mid \mu=0}^{*}-\partial_{\mu} v^{\infty}(0)\right)^{\mathrm{T}} \\
& =\left(\partial_{\mu} u_{\mid \mu=0}^{*}, \partial_{\mu} v_{\mid \mu=0}^{*}\right)^{\mathrm{T}}-\alpha(0) \in L_{\eta}^{2}\left(\mathbb{R}, \mathbb{C}^{2}\right),
\end{aligned}
$$

where $\alpha(\gamma)$ was defined in Lemma 5.10.

Step 2: Setup of the bifurcation problem. We choose $\eta>0$ small enough, but fixed, so that $\mathcal{L}$ is Fredholm on $L_{\eta, \text { even }}^{2}\left(\mathbb{R}, \mathbb{C}^{2}\right)$ by Lemma 5.8. Using Lemma 5.9, we have that ker $\mathcal{L}^{*}=$ $\operatorname{Span}\left\{(1,0)^{\mathrm{T}}\right\}$ on $L_{-\eta \text {,even }}^{2}\left(\mathbb{R}, \mathbb{C}^{2}\right)$, where again $\mathcal{L}^{*}$ refers to the $L^{2}$ adjoint. In particular,

$$
\operatorname{im} \mathcal{L}=\left\{(u, v)^{\mathrm{T}} \in L_{\eta, \text { even }}^{2}\left(\mathbb{R}, \mathbb{C}^{2}\right): \int_{\mathbb{R}} u(x) d x=0\right\}
$$

It follows that equation (5.14) with ansatz (5.15) is equivalent to the following system,

$$
\left\{\begin{array}{l}
F(w, \beta, \gamma)=0 \\
\left\langle\left(\mathcal{L}-\gamma^{2}\right)(w+\beta \alpha(\gamma) h(\gamma)),(1,0)^{\mathrm{T}}\right\rangle_{L^{2}}=0 .
\end{array}\right.
$$

Here,

$$
h(\gamma)=\chi_{+} e^{-\nu_{1}(\gamma)}+\chi_{-} e^{\nu_{1}(\gamma)},
$$

the function $F: H_{\eta \text {,even }}^{2}\left(\mathbb{R}, \mathbb{C}^{2}\right) \times \mathbb{C}^{2} \rightarrow \operatorname{im} \mathcal{L}$ is defined by

$$
F(w, \beta, \gamma)=P_{0}\left(\mathcal{L}-\gamma^{2}\right)(w+\beta \alpha(\gamma) h(\gamma))
$$

and $P_{0}$ is the orthogonal projection in $L_{\eta \text {,even }}^{2}\left(\mathbb{R}, \mathbb{C}^{2}\right)$ onto im $\mathcal{L}$. Summarizing, we view (5.17) as an equation $\mathcal{F}(w, \beta, \gamma)=0$ for the variables $(w, \beta, \gamma) \in H_{\eta \text {,even }}^{2}\left(\mathbb{R}, \mathbb{C}^{2}\right) \times \mathbb{C}^{2}$, with values $\mathcal{F} \in \operatorname{im} \mathcal{L} \times \mathbb{C}$. 
Step 3: Analyticity of $\mathcal{F}$. We first show that the map $\mathcal{F}_{0}: \gamma \rightarrow\left(\mathcal{L}-\gamma^{2}\right)(\alpha(\gamma) h(\gamma)) \in$ $L_{\eta}^{2}\left(\mathbb{R}, \mathbb{C}^{2}\right)$ is analytic in $B(0, \delta) \subset \mathbb{C}$ for some $\delta>0$. Let $\psi \in C^{\infty}(\mathbb{R})$ be a smooth, even function satisfying $\psi(x)=1$ for all $x \in[-1,1]$ and $\psi(x)=0$ for all $x \in \mathbb{R}$ with $|x| \geq 2$. We claim that

$$
\left(\mathcal{L}_{\infty}-\gamma^{2}\right)(\alpha(\gamma) h(\gamma))=\chi_{[-1,1]}\left(\mathcal{L}_{\infty}-\gamma^{2}\right)(\alpha(\gamma) \psi h(\gamma))
$$

where $\chi_{[-1,1]}$ is the characteristic function of the interval $[-1,1]$. Since the function defined by $\alpha(\gamma) e^{ \pm \nu_{1}(\gamma) x}$ satisfies (5.11) and $\psi^{\prime}(x)=\psi^{\prime \prime}(x)=0$ for all $x \in[-1,1]$ we have

$$
\left(\mathcal{L}_{\infty}-\gamma^{2}\right)(\alpha(\gamma) h(\gamma))(x)=0 \quad \text { for all } \quad x \in(-\infty,-1] \cup[1, \infty)
$$

Also, since $\mathcal{L}_{\infty}$ is a pointwise differential operator,

$$
\chi_{[-1,1]} \mathcal{L}_{\infty}(\psi u)=\chi_{[-1,1]} \mathcal{L}_{\infty} u \text { for all } u \in H_{\text {loc }}^{2}\left(\mathbb{R}, \mathbb{C}^{2}\right) .
$$

which together with (5.20) proves (5.19) and implies that

$$
\left(\mathcal{L}-\gamma^{2}\right)(\alpha(\gamma) h(\gamma))=\left(\mathcal{L}-\mathcal{L}_{\infty}\right)(\alpha(\gamma) h(\gamma))+\chi_{[-1,1]}\left(\mathcal{L}_{\infty}-\gamma^{2}\right)(\alpha(\gamma) \psi h(\gamma))
$$

Since $\mathcal{L}-\mathcal{L}_{\infty}$ is a second order differential operator whose matrix-valued coefficients decay exponentially at $\pm \infty$, we have that $\mathcal{L}-\mathcal{L}_{\infty}$ is bounded from $H_{-\eta, \text { even }}^{2}\left(\mathbb{R}, \mathbb{C}^{2}\right)$ to $L_{\eta, \text { even }}^{2}\left(\mathbb{R}, \mathbb{C}^{2}\right)$. Moreover, since $\psi \in C^{\infty}(\mathbb{R})$ has compact support the operator of multiplication by $\psi$ is bounded from $H_{-\eta \text {,even }}^{2}(\mathbb{R})$ to $H_{\eta \text {,even }}^{2}(\mathbb{R})$. We will show below that $h: B(0, \delta) \subset \mathbb{C} \rightarrow H_{-\eta \text {,even }}^{2}(\mathbb{R})$ is analytic. Then, using that $\alpha$ is analytic, and using (5.21), we conclude that $\mathcal{F}_{0}$ is analytic.

We define $h_{ \pm}(\gamma)(x):=e^{ \pm \nu_{1}(\gamma) x}$ so that we can express $h(\gamma)$ and its first- and second-order derivatives with respect to $x$ as linear combinations of products of $\chi_{ \pm}^{(k)}, \nu_{1}(\gamma)^{j}$ and $h_{ \pm}(\gamma)$, $k, j=0,1,2$. To show analyticity of $h(\gamma) \in H_{-\eta}^{2}(\mathbb{R})$, it is therefore enough to show that $h_{ \pm}: B(0, \delta) \subset \mathbb{C} \rightarrow L_{-\eta}^{2}(\mathbb{R})$ are analytic. Since $\nu_{1}$ is analytic and $\nu_{1}(0)=0$, we can choose $\delta>0$ small enough so that

$$
\left|\left(h_{ \pm}(\gamma)\right)(x)\right| \leq e^{(\eta / 2)|x|} \quad \text { for all } \quad x \in \mathbb{R}, \gamma \in B(0, \delta)
$$

This estimate implies that $h_{ \pm}(\gamma) \in L_{-\eta}^{2}(\mathbb{R})$ for all $\gamma \in B(0, \delta)$. It also implies, together with Lebesgue's Dominated Convergence Theorem, that $h_{ \pm}$is continuous. We next show that $h_{ \pm}$ is weekly analytic, that is, the function defined by $h_{ \pm}^{v}(\gamma):=\left\langle h_{ \pm}^{v}(\gamma), v\right\rangle_{L^{2}}$ is analytic for all $v \in L_{\eta}^{2}(\mathbb{R})$. This then implies that $h_{ \pm}$is actually analytic. To show this last step, we integrate $h_{ \pm}^{v}$ on the boundary of a rectangle $\mathrm{R} \subset B(0, \delta)$ using Fubini's Theorem. Since $e^{ \pm \nu_{1}(\gamma) x}$ is analytic in $\gamma$ for all $x \in \mathbb{R}$ we have

$$
\oint_{\partial \mathrm{R}} h_{ \pm}^{v}(\gamma) d \gamma=\int_{\mathbb{R}}\left(\oint_{\partial \mathrm{R}}\left(e^{ \pm \nu_{1}(\gamma) x}\right) d \gamma\right) v(x) d x=0
$$

This concludes the proof of analyticity of $\mathcal{F}_{0}$. It also shows that the map $\mathcal{F}$ is well defined as a map into $L_{\eta}^{2}\left(\mathbb{R}, \mathbb{C}^{2}\right)$. Moreover, since $\mathcal{F}$ is bounded linear in $w$ and $\beta$, and analytic in $\gamma$ by the above, we have analyticity of $\mathcal{F}$ as claimed. 
Step 4: Linearization and reduction. Equation (5.16) implies that $F\left(w_{0}, 1,0\right)=0$. We therefore have a trivial solution and can attempt to use the Implicit Function Theorem to find solutions nearby. Note that the linearization of the first equation in (5.17) in $w$ is

$$
F_{w}\left(w_{0}, 1,0\right)=P_{0} \mathcal{L}
$$

Lemmas 5.8 and 5.9 imply that $\mathcal{L}$ is Fredholm of index -1 with trivial kernel, and $P_{0}$ projects onto its range, so that the linearization in $w$ is boundedly invertible. Using the Implicit Function Theorem, we may therefore solve locally, and find a unique smooth solution

$$
w^{*}: B_{\delta}(1) \times B_{\delta}(0) \subset \mathbb{C}^{2} \rightarrow H_{\eta}^{2}\left(\mathbb{R}, \mathbb{C}^{2}\right), \quad w^{*}(1,0)=w_{0},
$$

so that locally

$$
F(w, \beta, \gamma)=0 \Longleftrightarrow w=w^{*}(\beta, \gamma) .
$$

Here $B_{\delta}\left(z_{0}\right) \subset \mathbb{C}$ is the open ball in $\mathbb{C}$, centered at $z_{0}$ of radius $\delta$. From this local uniqueness we conclude that $w^{*}(\beta, \gamma)=\beta w^{*}(1, \gamma)$ for all $(\beta, \gamma) \in B(1, \delta) \times B(0, \delta)$, so that we may restrict to $\beta=1$ in the sequel. By continuity of the solution, $w^{*}(1, \gamma)+\alpha(\gamma) h(\gamma) \neq 0$ for small $\gamma$.

Substituting $w^{*}$ into the second equation of (5.17) completes the Lyapunov-Schmidt reduction and gives us a bifurcation equation $E(\gamma)=0$, where $E: B_{\delta}(0) \subset \mathbb{C} \rightarrow \mathbb{C}$ is defined as

$$
E(\gamma)=\left\langle\left(\mathcal{L}-\gamma^{2}\right)\left(w^{*}(1, \gamma)+\alpha(\gamma) h(\gamma)\right),(1,0)^{\mathrm{T}}\right\rangle_{L^{2}}
$$

Since $w^{*}$ and the map $\mathcal{F}_{0}: \gamma \rightarrow\left(\mathcal{L}-\gamma^{2}\right)(\alpha(\gamma) h(\gamma))$ are analytic functions we conclude that $E$ is analytic.

Summarizing, for $\gamma>0, \gamma^{2}$ belongs to the point spectrum of $\mathcal{L}$ if and only if the reduced bifurcation problem $E(\gamma)=0$ possesses a solution. This proves the first statement of the proposition.

Step 5: Computing $E^{\prime}(0)$. Since $w^{*}(1, \gamma) \in H_{\eta, \text { even }}^{2}\left(\mathbb{R}, \mathbb{C}^{2}\right)$, from (5.23) we note

$$
E(\gamma)=\left\langle\mathcal{L}(\alpha(\gamma) h(\gamma)),(1,0)^{\mathrm{T}}\right\rangle_{L^{2}}+\mathcal{O}\left(\gamma^{2}\right)
$$

Expanding (5.18) in $\gamma$ and using the fact that $\chi_{+}+\chi_{-}=1$ we obtain

$$
\begin{aligned}
& (h(\gamma))(x)=1-\nu_{1}^{\prime}(0) \gamma x\left(\chi_{+}(x)-\chi_{-}(x)\right)+\mathcal{O}\left(\gamma^{2}\right) \\
& (h(\gamma))^{\prime}(x)=-\nu_{1}^{\prime}(0) \gamma\left(\chi_{+}(x)-\chi_{-}(x)\right)-\nu_{1}^{\prime}(0) \gamma x\left(\chi_{+}^{\prime}(x)-\chi_{-}^{\prime}(x)\right)+\mathcal{O}\left(\gamma^{2}\right) .
\end{aligned}
$$

Let $l_{1}=a_{u}^{*} u_{x}^{*}+b_{u}^{*} v_{x}^{*}, l_{2}=a_{v}^{*} u_{x}^{*}+b_{v}^{*} v_{x}^{*}$ and let $k_{1}(\cdot, \gamma)$ be the first component of $\mathcal{L}(\alpha(\gamma) h(\gamma))$. We now compute $k_{1}(x, \gamma)$, expanding in $\gamma$. We first use the zeroth order expansion of $\alpha(\gamma)$ from Lemma 5.10, and then the expansion of $h$ obtained above, in the first row of $\mathcal{L}$ defined in (3.2), to obtain

$$
k_{1}(x, \gamma)=\partial_{x}\left[\left(f_{u}^{\infty} b^{*}(x)-f_{v}^{\infty} a^{*}(x)+\mathcal{O}(\gamma)\right)(h(\gamma))^{\prime}(x)\right.
$$




$$
\begin{gathered}
\left.+\left(f_{u}^{\infty} l_{2}(x)-f_{v}^{\infty} l_{1}(x)+\mathcal{O}(\gamma)\right)(h(\gamma))(x)\right] \\
=\partial_{x}\left[\left(f_{v}^{\infty} a^{*}(x)-f_{u}^{\infty} b^{*}(x)\right) \nu_{1}^{\prime}(0) \gamma\left(\chi_{+}(x)-\chi_{-}(x)\right)+\Theta(x, \gamma)+\mathcal{O}\left(\gamma^{2}\right)\right] .
\end{gathered}
$$

Here, $\Theta(x, \gamma)$ is a finite sum whose terms contain one of the functions $l_{1}(x), l_{2}(x)$ and $x\left(\chi_{+}^{\prime}(x)-\right.$ $\left.\chi_{-}^{\prime}(x)\right)$. Therefore, we have $\Theta(x, \gamma) \rightarrow 0$ as $|x| \rightarrow \infty$. Taking the scalar product with the constant $(1,0)^{\mathrm{T}}$ gives the desired expansion for $E$,

$$
E(\gamma)=\int_{\mathbb{R}} k_{1}(x, \gamma) d x=2\left(a^{\infty} f_{v}^{\infty}-b^{\infty} f_{u}^{\infty}\right) \nu_{1}^{\prime}(0) \gamma+\mathcal{O}\left(\gamma^{2}\right)
$$

Using the expansion for $\nu_{1}^{\prime}(0)$ from Lemma 5.10, we immediately arrive at (5.13).

Step 6: Invertibility for $E(\gamma) \neq 0$. We conclude the proof by showing that eigenfunctions are necessarily of the form described in the ansatz (5.15). Equivalently, we can show that $\mathcal{L}-\lambda$ is invertible for $\operatorname{Re} \lambda>0$ whenever $E(\gamma) \neq 0$. We therefore need to solve

$$
\left(\mathcal{L}-\gamma^{2}\right)\left(\begin{array}{l}
u \\
v
\end{array}\right)=\left(\begin{array}{l}
r_{1} \\
r_{2}
\end{array}\right)
$$

for right-hand sides $r_{1}, r_{2} \in L^{2}\left(\mathbb{R}, \mathbb{C}^{2}\right)$. Note that $\mathcal{L}-\gamma^{2}$ is Fredholm of index 0 since $\gamma^{2}$ is not in the essential spectrum for $\operatorname{Re} \lambda>0$. It is therefore sufficient to solve this equation for right-hand sides $r_{j}$ in a dense subset of $L^{2}\left(\mathbb{R}, \mathbb{C}^{2}\right)$, which we chose to be $L_{\eta}^{2}\left(\mathbb{R}, \mathbb{C}^{2}\right)$. We look for solutions to this equation in the form of our ansatz (5.15), which for $\operatorname{Re} \lambda>0$ clearly provides us with $L^{2}$-functions. We end up with the equation

$$
\left\{\begin{array}{l}
F(w, \beta, \gamma)=P_{0}\left(r_{1}, r_{2}\right)^{\mathrm{T}} \\
\left\langle\left(\mathcal{L}-\gamma^{2}\right)(w+\beta \alpha(\gamma) h(\gamma)),(1,0)^{\mathrm{T}}\right\rangle_{L^{2}}=\left\langle 1, r_{1}\right\rangle_{L^{2}}
\end{array}\right.
$$

This is a linear equation in $w$ and $\beta$, and the joint linearization is Fredholm of index zero, by Step 4 . We can therefore conclude that we can solve this equation with bounded inverse provided that there is no kernel, which amounts precisely to the condition $E(\gamma) \neq 0$. This proves the proposition.

Remark 5.12. Proposition 5.11 is valid for all $\tau \in[0,1]$, and the function $E$ is smoothly depending on $\tau$. This can be readily seen from the smoothness of eigenfunctions and eigenvectors of the asymptotic problem and smoothness of the reduction procedure.

\subsection{Proof of Proposition 4.1}

We are now ready to put together the ingredients for the proof of Proposition 4.1. Proposition 5.11 implies that for any $\tau \in[0,1]$, there exists $\delta_{\tau}>0$ and an analytic function $E_{\tau}: B_{\delta_{\tau}}(0) \subset \mathbb{C} \rightarrow \mathbb{C}$ that detects the eigenvalues of $\mathcal{L}_{\tau}$ according to (5.12). Since the 
derivative of $E_{\tau}$ does not vanish at the origin, Proposition 5.11, we conclude that $\delta_{\tau}>0$ can be chosen small enough so that $E_{\tau}(\gamma) \neq 0$ for any $\gamma \in B_{\delta_{\tau}}(0)$. Thus, $\mathcal{L}_{\tau}$ has no eigenvalues in $\left(0, \delta_{\tau}\right)$. By Remark 5.12, $E$ is smooth in $\tau$, which implies that we can find $0<\delta^{*}<\delta_{\tau}$ for all $\tau \in[0,1]$. In addition, equation (3.1) is well-posed for all $\tau$, which implies $\sup \operatorname{Re} \sigma\left(\mathcal{L}_{\tau}\right)<\infty$. Thus, the number of real unstable eigenvalues of $\mathcal{L}$ is finite,

$$
N(\tau)=\#\left\{\lambda(\tau) \in \sigma_{\text {point }}\left(\mathcal{L}_{\tau}\right): \lambda(\tau)>0\right\}<\infty
$$

for all $\tau \in[0,1]$. We can therefore define a parity index as follows,

$$
i_{\mathrm{p}}(\tau)=(-1)^{N(\tau)}
$$

Since $\mathcal{L}_{\tau}$ has no eigenvalues in $\left(0, \delta^{*}\right)$ or in $\left(1 / \delta^{*}, \infty\right)$, for $\delta^{*}$ sufficiently small, eigenvalues can leave the positive semi-axis only in pairs by becoming complex; see also Figure 2. Hence, the parity index is constant during the homotopy. Finally, Lemma 5.3 shows that $i_{p}(1)=-1$. Therefore $i_{p}(0)=-1$ and we can conclude that $\mathcal{L}$ has at least one positive eigenvalue. This proves Proposition 4.1 and thereby concludes the proof of Theorem 1.1.

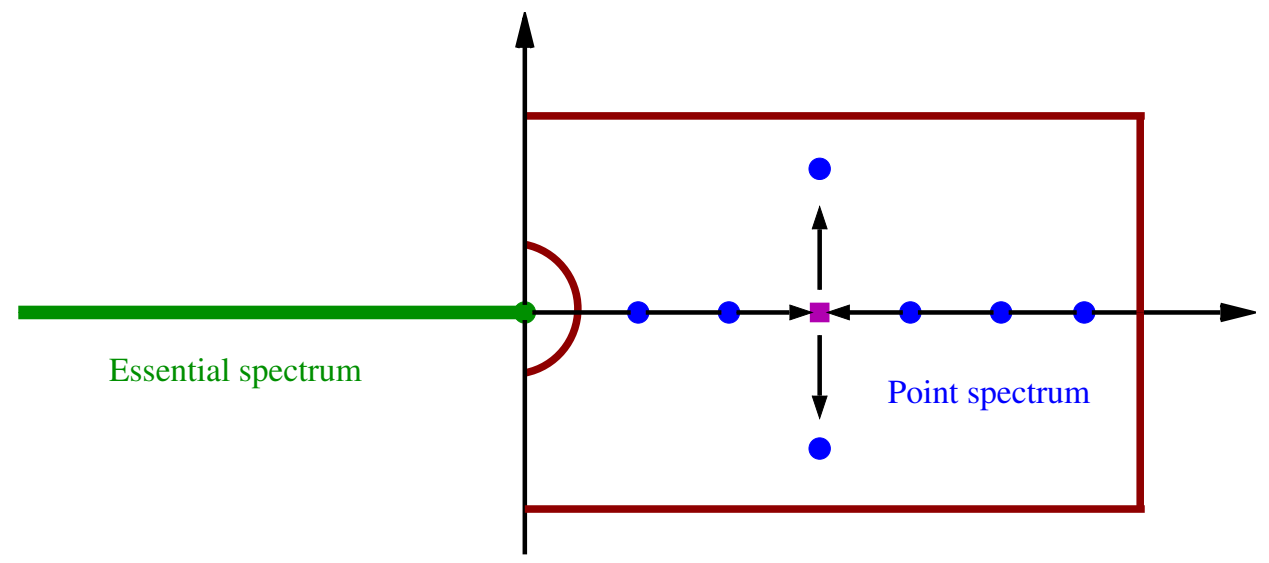

Figure 2: A schematic plot of eigenvalue movement during the homotopy: eigenvalues cannot cross the upper and right boundary of the rectangle (brown in color), so $i_{p}(\tau) \equiv-1$ and the unstable dimension only changes through Hopf bifurcations. Essential spectrum $\sigma_{\text {ess }}\left(\mathcal{L}_{\tau}\right)$ is the located on the negative real axis (red in color plot), point spectrum $\sigma_{\text {point }}\left(\mathcal{L}_{\tau}\right)$ are bullets in the unstable complex plane (blue in color).

\section{Examples}

Examples of spikes in systems of the type (1.1) are plentiful. In soms cases spike solutions are explicitly given. Consider for example the simplest prototype of a chemical conversion reaction of the type (1.2) is

$$
\left\{\begin{array}{c}
p_{t}=p_{x x}-g(p, q), \\
q_{t}=d q_{x x}+g(p, q),
\end{array} \quad t \geq 0, x \in \mathbb{R} .\right.
$$


with $g(p, q)=-p-q+p^{2}$ and where $d$ is small. Adding the two equation, one finds $p+d q=\mu$ is constant in $x$ for equilibria, so that

$$
p_{x x}+\frac{\mu+(d-1) p}{d}-p^{2}=0 .
$$

This family possesses the explicit family of spike solutions

$$
\begin{aligned}
& p^{*}(x)=\frac{d-1}{2 d}+\zeta(\mu)\left(1-3 \operatorname{sech}^{2}\left(x \sqrt{\frac{\zeta(\mu)}{2}}\right)\right), \\
& q^{*}(x)=\frac{\mu}{d}-\frac{d-1}{2 d^{2}}-\frac{\zeta(\mu)}{d}\left(1-3 \operatorname{sech}^{2}\left(x \sqrt{\frac{\zeta(\mu)}{2}}\right)\right),
\end{aligned}
$$

where

$$
\zeta(\mu)=\frac{\sqrt{(d-1)^{2}+4 \mu d}}{2 d} .
$$

Note that $\zeta(\cdot)$ is an analytic function in a neighborhood of 0 . More realistic examples for conversion reactions contain threshold kinetics

$$
g(p, q)=\delta q(1-q)(q-\alpha)+p \quad \text { or } \quad g(p, q)=\delta q(1-q)\left(q-\frac{\alpha-p}{\alpha}\right)+\kappa,
$$

where $\alpha$ is a threshold in the bistable $q$-kinetics. Again, one readily finds spikes after adding both equations. In the first case, writing $u=p+d q$ and $\varphi=q$, one obtains a scaled version of the phase-field model (1.3). Both models have also been successfully used to model precipitation kinetics as observed in Liesegang patterns [4].

Phase-field models (1.3) contain the Cahn-Hilliard equation

$$
u_{t}=-\partial_{x x}\left(\partial_{x x} u+u-u^{3}\right), t \geq 0, x \in \mathbb{R}
$$

as a formal limit for infinite latent heat, $l \rightarrow \infty$. In fact, our arguments can be used to establish instability of spikes for the Cahn-Hilliard equation using a homotopy through the phase-field system. More precisely, consider the family of equations

$$
\left(\begin{array}{cc}
\tau & 0 \\
1-\tau & \tau
\end{array}\right)\left(\begin{array}{c}
u_{t} \\
v_{t}
\end{array}\right)=\left(\begin{array}{cc}
\partial_{x}^{2} & 1 \\
0 & \partial_{x}^{2}
\end{array}\right)\left(\begin{array}{l}
u \\
v
\end{array}\right)+\left(\begin{array}{c}
u-u^{3} \\
0
\end{array}\right) t \geq 0, x \in \mathbb{R} .
$$

For $\tau=0$, we find the Cahn-Hilliard equation, and for $\tau=1$, we find the Allen-Cahn equation coupled with source term from a decoupled heat equation. For $\tau \in(0,1)$, we have a scaled version of the phase field system. Equilibria are clearly independent of $\tau$ and one finds families of spikes. Since those are unstable at $\tau=1$ with precisely one unstable eigenvalue, one is tempted to conclude instability for all values of $\tau$. This can indeed be made rigorous by employing the very same techniques used in the present paper. One checks that the essential spectrum of the linearization at a spike remains in the closed left half plane $\operatorname{Re} \lambda \leq 0$ for all values of $\tau$, provided this is true at $\tau=0$. In fact, the essential spectrum is always stable, equal to $(-\infty, 0]$, for this class of models provided a spike exists. One also readily verifies that $E^{\prime}(0) \neq 0$ for all $\tau$, which shows that the parity is negative for all $\tau$ and proves instability. We note that instability of 
spikes on unbounded domains was proved in [10]. We are not aware of an explicit reference for instability of spikes in phase field models.

Lastly, we mention the Keller-Segel model,

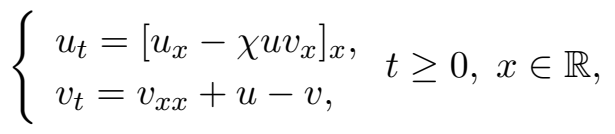

One solves for $u=\mu \mathrm{e}^{\chi^{v}}$ and then solves

$$
0=v_{x x}+\mu \mathrm{e}^{\chi v}-v
$$

which possesses spikes for $\mu<(\mathrm{e} \chi)^{-1}$.

\section{Discussion}

Our results show that spikes are always unstable with respect to an even, localized eigenfunction to a positive real eigenvalue. We do not know when this eigenfunction possesses more structure, such as a sign in the $v$-component beyond $\tau=1$.

On the other hand, one often does observe spikes in experiments and numerical simulations. There are two aspects to this apparent discrepancy between theory and observation.

First, unstable eigenvalues can be very small. This happens in particular when spikes are formed by pairs of layers that interact weakly. Examples of such type are readily constructed in the bistable models (6.2), with steady-state equation $u_{x x}+u-u^{3}+v=0$, with $v \equiv \mu$ constant. For $\mu \sim 0$, spikes form long plateaus, and the unstable eigenvalue converges to zero as $\mu \rightarrow 0$.

Secondly, our instability results are valid only for infinite domains. Imposing Neumann boundary conditions at $x= \pm L$, for instance, will change the spectrum. Convergence results for the point and essential spectrum [18] show that the spectrum converges as a set for $L \rightarrow \infty$. Moreover, the convergence of point eigenvalues is exponential in $L$, so that for only moderate sizes of the domain, our results give instability of spikes. The caveat here is that this instability is not uniform in the family of spikes, so that spikes of large amplitude (as in chemotaxis models) or large width (as in phase field models) may well be stable in finite domains of large size.

Finally, we point out a few open questions, some of which are subject of future work.

We expect that our instability results carry over to higher space dimensions. Again, the existence problem is well studied and spikes are radially symmetric. At the end of our homotopy, $\tau=1$, spikes are unstable in the space of radially symmetric functions and one expects this instability to persist during the homotopy.

Our arguments also apply to layers, that is solutions $\left(u^{*}, v^{*}\right)$ that converge to different limits $\left(u^{ \pm}, v^{ \pm}\right)$as $x \rightarrow \pm \infty$. In the scalar case, and at the end of the homotopy, these are stable solutions to the PDE. Our method then readily gives that the number of unstable eigenvalues for such layers is even, $i_{\mathrm{p}}=1$. Inspecting the argument in Section 5.7, one sees that layers can destabilize during the homotopy only when Hopf bifurcation occurs. This can often be 
excluded when Lyapunov functions are known to exist, such as for the Keller-Segel model or the phase-field system. We do not know if Hopf bifurcations from layers can occur in our more general class of conservation laws coupled to a reaction-diffusion equation.

\section{References}

[1] G. Caginalp, An analysis of a phase field model of a free boundary, Arch. Ration. Mech. Anal. 92 (1986), 205-245.

[2] M. Droz, Recent theoretical developments on the formation of Liesegang patterns, Jour. Stat. Phys. 101 (2000), 509-519.

[3] R. A. Gardner, K. Zumbrun, The Gap Lemma and Geometric Criteria for Instability of Viscous Shock Profiles, Comm. Pure Appl. Math. 51 (1998), 797-855.

[4] R. Goh, S. Mesuro, A. Scheel, Threshold models for precipitation and Liesegang patterns, in preparation.

[5] P. S. Hagan, The instability of nonmonotonic wave solutions of parabolic equations, Stud. Appl. Math. 64 (1981), no. 1, 57-88.

[6] D. Henry, Geometric Theory of Semilinear Parabolic Equations, Lect. Notes Math. 840, Springer-Verlag, New York, 1981.

[7] D. Hilhorst, R. van der Hout, M. Mimura, I. Ohnishi, Fast reaction limits and Liesegang bands. Free boundary problems, 241-250, Internat. Ser. Numer. Math., 154 (2007), Birkhuser, Basel.

[8] T. Hillen, K. Painter, A User's Guide to PDE Models for Chemotaxis, J. Math. Biol., 58(1), (2008), 183-217.

[9] T. Hillen, A. Potapov, The One-Dimensional Chemotaxis Model: Global Existence and Asymptotic Profile, Math. Meth. Appl. Sci., 27 (2004), 1783-1801.

[10] P. Howard, Spectral analysis of stationary solutions of the Cahn-Hilliard equation, Adv. Diff. Eq. 14 (2009) 87-120.

[11] A. Jilkine, L. Keshet, Y. Mori, Wave-pinning and Cell Polarity from a Bistable Reactiondiffusion System Bioph. J., 94 (2008), 3684-3697.

[12] T. Kapitula, B. Sandstede, Stability of Bright SolitaryWave Solutions to Perturbed Nonlinear Schrödinger Equations, Physica D 124 (1998), 58-103.

[13] E. Keller and L. Segel, Initiation of slime mold aggregation viewed as an instability, J. Theor. Biol. 26 (1970), 399-415.

[14] J. Keller, S. Rubinow, Recurrent precipitation and Liesegang rings, J. Chem. Phys. 74 (1981), no. 9, 5000-5007. 
[15] A. Lunardi, Analytic semigroups and optimal regularity in parabolic problems, Birkhuser Verlag, Basel (1995).

[16] K. J. Palmer, Exponential dichotomies and transversal homoclinic points, J. Diff. Eq. 55 (1984), 225-256.

[17] K. J. Palmer, Exponential dichotomies and Fredholm operators, Proc. Amer. Math. Soc. 104 (1988), 149-156.

[18] B. Sandstede and A. Scheel, Absolute and convective instabilities in bounded domains, Physica D 145 (2000), 233-277.

[19] B. Sandstede and A. Scheel, Relative Morse indices, Fredholm indices, and group velocities, Discr. Cont. Dynam. Syst., 20 (2008), 139-158.

[20] A. Scheel, Robustness of Liesegang patterns, Nonlinearity 22 (2009), 457-483. 